\title{
COINCIDENCE AND COMMON FIXED POINTS OF HYBRID CONTRACTIONS
}

\author{
M. S. KHAN, Y. J. CHO, W. T. PARK and T. MUMTAZ
}

(Received 10 January 1991; revised 15 April 1991)

Communicated by J. H. Rubinstein

\begin{abstract}
In this paper, we show the existence of solutions of functional equations $f_{i} x \in S x \cap T x$ and $x=f_{i} x \in S x \cap T x$ under certain nonlinear hybrid contraction and asymptotic regularity conditions, generalize and improve a recent result due to Kaneko concerning common fixed points of multivalued mappings weakly commuting with a single-valued mapping and satisfying a generalized contraction type. Some related results are also obtained.
\end{abstract}

1991 Mathematics subject classification (Amer. Math. Soc.): 54 H 25.

Keywords and phrases: Coincidence and common fixed points, Hausdorff metric, orbital complete, proximinal subsets and weakly commuting mappings.

\section{Introduction}

Nadler [12] proved a fixed point theorem for multi-valued contraction mappings, which is called Nadler's contraction principle. Subsequently, a number of generalization of Nadler's contraction principle were obtained by Ciric [23], Khan [8], Kubiak [9], Kaneke [6, 7], Sessa [14, 19], Singh [10] and many others $[22,24]$.

Recently, non-linear hybrid contractions, that is, contraction types involving single-valued and multi-valued mappings have been studied by Mukherjee [11], Naimpally et al. [13], Rhoades et al. [16] and Sessa et al. [20, 19].

In this paper, we show the existence of solutions of functional equations

(C) 1993 Australian Mathematical Society 0263-61 15/93 \$A2.00+0.00 
$f_{i} x \in S x \cap T x$ and $x=f_{i} \in S x \cap T x$ under certain non-linear hybrid contraction and asymptotic regularity conditions where $\left\{f_{i}\right\}$ is a family of single-valued mappings on a metric space, $S$ and $T$ are multi-valued mappings on a metric space. Our results are generalizations and improvements of some results due to Kaneko, Kubiak, Mukherjee, Naimpally et al., Rhoades et al. and many others. Also, we obtain other related results by using the proximinality of sets.

\section{Preliminaries}

Let $(X, d)$ be a metric space. A subset $K$ of $X$ is said to be proximinal if for each $x \in X$, there exists a point $y \in K$ such that $d(x, y)=D(x, K)$, where $D(x, A)$ denotes the ordinary distance between $x \in X$ and a non-empty subset $A$ of $X$. We shall use the following notation and definitions:

$C L(X)=\{A: A$ is a non-empty closed subset of $X\}$,

$C B(X)=\{A: A$ is a non-empty closed and bounded subset of $X\}$,

$C(X)=\{A: A$ is a non-empty compact subset of $X\}$ and

$P_{x}(X)=\{A: A$ is a non-empty proximinal subset of $X\}$.

For $A, B \in C L(X)$ and $\varepsilon>0$.

$$
\begin{aligned}
N(\varepsilon, A) & =\{x \in X: d(x, a)<\varepsilon \text { for some } a \in A\}, \\
E_{A, B} & =\{\varepsilon>0: A \subseteq N(\varepsilon, B) \text { and } B \subseteq N(\varepsilon, A)\}, \text { and } \\
H(A, B) & = \begin{cases}\inf E_{A, B}, & \text { if } E_{A, B} \neq \emptyset \\
+\infty, & \text { if } E_{A, B}=\emptyset .\end{cases}
\end{aligned}
$$

$H$ is called the generalized Hausdorff distance function for $C L(X)$ induced by the metric $d$, and $H$ defined on $C B(X)$ is said to be the Hausdorff metric induced by $d$.

It is well-known that $P_{x}(X) \subset C L(X)$ and $C(X) \subset P_{X}(X)$ ([21]).

Let $\left\{f_{i}\right\}$ be a family of a singled-valued mappings from $X$ to itself and $S, T$ be multi-valued mappings from $X$ to the non-empty subsets of $X$.

DEFINITION 2.1. If, for $x_{0} \in X$, there exist sequences $\left\{x_{n}\right\}$ and $\left\{y_{n}\right\}$ in $X$ such that

$$
\begin{aligned}
y_{2 n} & =f_{2 n} x_{2 n-1} \in S x_{2 n} \quad \text { for every } n \in \mathbb{N}, \\
y_{2 n+1} & =f_{2 n+1} x_{2 n} \in T x_{2 n+1} \quad \text { for every } n \in \mathbb{N}_{0}=\mathbb{N} \cup\{0\},
\end{aligned}
$$


then $0_{f_{i}}\left(x_{0}\right)=\left\{y_{n}: n=0,1,2, \ldots\right\}$ is said to be the orbit for $\left(S, T ; f_{i}\right)$ at $x_{0}$. Further, $0_{f_{i}}\left(x_{0}\right)$ is called a regular orbit for $(S, T ; f)$ if

$$
d\left(y_{n}, y_{n+1}\right) \leq \begin{cases}H\left(S x_{n-1}, T x_{n}\right), & \text { if } n \text { is odd } \\ \left.H\left(T x_{n-1}\right), S x_{n}\right), & \text { if } n \text { is even }\end{cases}
$$

DEFINITION 2.2. If, for $x_{0} \in X$, there exist sequences $\left\{x_{n}\right\}$ and $\left\{y_{n}\right\}$ in $X$ such that every Cauchy sequence of the form $o_{f_{i}}\left(x_{0}\right)$ converges in $X$, then $X$ is called $\left(S, T ; f_{i}\right)$-orbitally complete with respect to $x_{0}$ or simply $\left(S, T ; f_{i}, x_{0}\right)$-orbitally complete.

If, for each $i \in \mathbb{N}, f_{i}$ is an identity mapping on $X$, then $0_{f_{i}}\left(x_{0}\right)$ is denoted by $O\left(x_{0}\right)$ and $\left(S, T ; f_{i}, x_{0}\right)$-orbitally completeness by $\left(S, T ; x_{0}\right)$-orbital completeness.

DEFINITION 2.3. A pair $(S, T)$ is said to be asymptotically regular at $x_{0} \in X$ if for any sequence $\left\{x_{n}\right\}$ in $X$ and each sequence $\left\{y_{n}\right\}$ in $X$ such that $y_{n} \in$ $S x_{n-1} \cup T x_{n-1}, \lim _{n \rightarrow \infty} d\left(y_{n}, y_{n+1}\right)=0$.

We remark that Definitions 2.1-2.3 with $S=T$ and $f_{i}=f$ for each $i \in \mathbb{N}$ reduce to Definitions 4, 6 and 7 of [16], respectively, and orbital completeness need not imply the completeness of the space. Evidently, a complete space is orbitally complete.

DEFINITION 2.4. For each $i \in \mathbb{N}$, the mappings $f_{i}$ and $S$ are said to be commuting at a point $x \in X$ ([2]) if $f_{i} S x \subseteq S f_{i} x$. The mappings $f_{i}$ and $S$ are said to be commuting on $X$ if $f_{i} S x \subseteq S f_{i} x$ for every point $x \in X$.

In [17], Sessa introduced the concept of weak commutativity for single-valued mappings on a metric space and Sessa et al. [19] extended this concept to the setting of a single-valued mapping and a multi-valued mapping on a metric space.

DEFINITION 2.5. For each $i \in \mathbb{N}$, the mappings $f_{i}$ and $S$ are said to be weakly commuting at $x \in X$ if $H\left(f_{i} S x, S f_{i} x\right) \leq D\left(f_{i} x, S x\right)$. The mapping $f$ and $S$ are said to be weakly commuting on $X$ if they are weakly commuting at every $x \in X$.

Note that commutativity implies weak commutativity, but the converse need not be true even in the case of singled-valued mappings as shown in Section 4. 
EXAMPLE. Let $x=\{1,2,3,4\}$, define a metric $d$ on $x$ and mappings $f, S$ on $X$ as follows:

$$
\begin{aligned}
d(1,2) & =d(3,4)=2, & d(1,3) & =d(2,4)=1 \\
d(1,4) & =d(2,3)=3 / 2, & & \\
S(1) & =S(3)=\{4\}, & S(2) & =S=S(4)=\{3\}, \\
f(1) & =f(2)=f(4)=2, & f(4) & =1, \text { respectively. }
\end{aligned}
$$

Then we have $S f(1)=\{3\}$ and $f S(1)=\{1\}$ and so $f$ and $S$ do not commute at $x=1$, but $f$ and $S$ are weakly commuting at $x=1$ since

$$
H(S f(1), f S(1))=D(f(1), S(1))=2 .
$$

Let $\mathscr{F}$ be the family of all mappings $\phi$ from the set $R^{+}$of non-negative real numbers to itself such that $\emptyset$ is upper-semicontinuous, non-decreasing and $\phi(t)<t$ for any $t>0$.

The following theorem is an interesting result for the existence of coincidence points of non-linear hybrid contractions:

THEOREM 2.1. [16] Let $T$ be a multi-valued mapping from a metric space $X$ into $C L(X)$. If there exists a mapping from $X$ into itself such that $T(X) \subseteq$ $f(X)$, for each $x, y \in X$ and $\phi \in \mathscr{F}$,

(2.3) there exists a point $x_{0} \in X$ such that $T$ is asymptotically regular at $x_{0}$ and $f(X)$ is $\left(T ; f, x_{0}\right)$-arbitrally complete, then $f$ and $T$ have a coincidence point in $X$.

If $f$ is not the identity mapping, then the commuting mappings $f$ and $T$ satisfying the hypotheses of Theorem 2.1 need not have a common fixed point in $X$.

Now we can consider: What additional conditions will guarantee the existence of a common fixed point of $f$ and $T$ ?

In [16], Rhoades et al. gave the solution to this problem. In this paper, we also investigate different sets of conditions under which the fixed point equation

$$
x=f_{i} x \in S x \cap T x \quad \text { for } x \in X \text { and each } i \in \mathbb{N}
$$

possesses a solution. 


\section{Results}

Now we are ready to give our main theorems:

THEOREM 3.1. Let $S$ and $T$ be multi-valued mappings from a metric space $X$ into $P_{X}(x)$ and let $\left\{f_{i}\right\}$ be the family of all continuous mappings from $X$ into itself such that

$$
\begin{aligned}
& \text { (3.2) } \quad H(S x, T x) \leq \\
& \phi\left(\max \left(D\left(f_{i} x, S x\right), D\left(f_{j} y, T y\right), D\left(f_{i} x, T y\right), D\left(f_{j} y, S x\right), d\left(f_{i} x, f_{j} y\right)\right)\right) \\
& \quad \text { for each } x, y \in X, i, j \in \mathbb{N}, i \neq j \text { and } \phi \in \mathscr{F}, \\
& \quad \phi(t) \leq q \text { for each } t>0 \text { and for some fixed } q \in(0,1), \\
& \text { (3.3) } \quad \text { there exists a point } x_{0} \in X \text { such that the pair }(S, T) \text { is asymptot- } \\
& \text { ically regular at } x_{0}, \text { and } \\
& \text { (3.5) for each } i \in \mathbb{N}, f_{i}(X) \text { is }\left(S, T ; f_{i}, x_{0}\right) \text {-orbitally complete. }
\end{aligned}
$$

Then (1) $f_{i}, S$ and $T$ have a coincidence point in $X$.

If $z$ is a coincidence point of $f_{i}, S$ and $T$ and $f_{i} z$ is a fixed point of $f_{i}$, then we have:

(2) $f_{i} z$ is also a fixed point of $S$ provided $f_{i}$ is weakly commuting with $S$ at $z$ for every even $i \in \mathbb{N}$ for any odd $j \in \mathbb{N}$;

(3) $f_{i} z$ is also a fixed point of $T$ provided $f_{i}$ is weakly commuting with $T$ at $z$

(4) $f_{i} z$ is a common fixed point of $S$ and $T$ provided $f_{i}$ is weakly commuting with each of $S$ and $T$ at $z$.

PROOF. (1) Let $x_{0}$ be a point in $X$ satisfying (3.4). Since $S(X) \cup T(X) \subset$ $f_{i}(X)$, for each $i \in \mathbb{N}$, we can find sequences $\left\{x_{n}\right\}$ and $\left\{y_{n}\right\}$ in $X$ such that

$$
\begin{aligned}
y_{2 n} & =f_{2 n} x_{2 n-1} \in S x_{2 n} \quad \text { for every } n \in \mathbb{N}, \\
y_{2 n+1} & =f_{2 n+1} x_{2 n} \in T x_{2 n+1} \quad \text { for every } n \in \mathbb{N}_{0}, \\
d\left(y_{2 n}, y_{2 n+1}\right) & \leq q^{-1 / 2} H\left(S x_{2 n}, T x_{2 n+1}\right) \quad \text { and } \\
d\left(y_{2 n+1}, y_{2 n+2}\right) & \leq q^{-1 / 2} H\left(T x_{2 n+1}, S x_{2 n+2}\right) .
\end{aligned}
$$

By (3.4), we have $\lim _{n \rightarrow \infty} d\left(y_{n}, y_{n+1}\right)=0$.

Now, we want to show that $\left\{y_{2 n}\right\}$ is a Cauchy sequence in $f_{i}(X)$. Suppose that the sequence $\left\{y_{2 n}\right\}$ is not a Cauchy sequence. Then there exists a positive 
number $\varepsilon$ such that, for each positive integer $\kappa$, there exist integers $n(\kappa)$ and $m(k)$ such that

$$
\kappa \leq n(\kappa)<m(\kappa)
$$

and

$$
d\left(y_{2 n(\kappa)}, y_{2 m(\kappa)}\right) \geq \varepsilon .
$$

Let $d_{i, j}=d\left(y_{i}, y_{j}\right)$ and $d_{i}=d\left(y_{i}, y_{i+1}\right)$ for each $i, j \in \mathbb{N}$. Then, for each integer $\kappa$, we have

$$
\varepsilon \leq d_{2 n(\kappa), 2 m(\kappa)} \leq d_{2 n(\kappa), 2 m(\kappa)-2}+d_{2 m(\kappa)-2}+d_{2 m(\kappa)-1}
$$

For each integer $\kappa$, let $m(\kappa)$ denote the smallest integer satisfying (3.a) and (3.b) for some $n(\kappa)$. Then we have $d_{2 n(\kappa), 2 m(\kappa)-2}<\varepsilon$ and it follows from (3.c) that

$$
\lim _{\kappa \rightarrow \infty} d_{2 n(\kappa), 2 m(\kappa)}=\varepsilon .
$$

Using the triangle inequality, we get

$$
\begin{aligned}
\left|d_{2 n(\kappa), 2 m(\kappa)-1}-d_{2 n(\kappa), 2 m(\kappa)}\right| & \leq d_{2 m(\kappa)-1} \quad \text { and } \\
\left|d_{2 n(\kappa)+1,2 m(\kappa)-1}-d_{2 n(\kappa), 2 m(\kappa)}\right| & \leq d_{2 n(\kappa)}+d_{2 m(\kappa)-1},
\end{aligned}
$$

which yield

$$
\lim _{\kappa \rightarrow \infty} d_{2 n(\kappa), 2 m(\kappa)-1}=\lim _{\kappa \rightarrow \infty} d_{2 n(\kappa)+1,2 m(\kappa)-1}=\varepsilon
$$

in view of (3.4) and (3.d) and so, by (3.2), we have

$$
\begin{aligned}
d_{2 n(\kappa), 2 m(\kappa)} \leq & d_{2 n(\kappa)}+d_{2 n(\kappa)+1,2 m(\kappa)} \\
\leq & d_{2 n(\kappa)}+q^{-1 / 2} H\left(S x_{2 m(\kappa)}, T x_{2 n(\kappa)+1}\right) \\
\leq & d_{2 n(\kappa)}+q^{-1 / 2} \phi\left(\operatorname { m a x } \left(D\left(f_{2 m(\kappa)+1} x_{2 m(\kappa)}, S x_{2 m(\kappa)}\right),\right.\right. \\
& D\left(f_{2 n(\kappa)+2} x_{2 n(\kappa)+1}, T x_{2 n(\kappa)+1}\right), D\left(f_{2 n(\kappa)+2} x_{2 n(\kappa)+1}, S x_{2 m(\kappa)}\right), \\
& \left.D\left(f_{2 m(\kappa)+1} x_{2 m(\kappa)}, T x_{2 n(\kappa)+1}\right), d\left(f_{2 m(\kappa)+1} x_{2 m(\kappa)}, f_{2 n(\kappa)+2} x_{2 n(\kappa)+1}\right)\right), \\
\leq & d_{2 n(\kappa)}+q^{-1 / 2} \phi\left(\operatorname { m a x } \left(d_{2 m(\kappa)}, d_{2 n(\kappa)+1}, d_{2 n(\kappa)+2,2 m(\kappa)},\right.\right. \\
& \left.\left.d_{2 n(\kappa)+1,2 m(\kappa)+1}, d_{2 n(\kappa)+2,2 m(\kappa)+1}\right)\right) .
\end{aligned}
$$

Using the upper semicontinuity of $\phi$ and letting $\kappa \rightarrow \infty$, this inequality yields

$$
\varepsilon \leq q^{-1 / 2} \phi(\varepsilon) \leq q^{-1 / 2} q \varepsilon<\varepsilon
$$


since $\varepsilon>0$ and $q^{1-1 / 2}<1$, which contradicts the choice of $\varepsilon$ and so the sequence $\left\{y_{2 n}\right\}$ is a Cauchy sequence. Similarly, we can prove that $\left\{y_{2 n+1}\right\}$ is also a Cauchy sequence in $f_{i}(X)$.

Since $f_{i}(X)$ is $\left(S, T ; f_{i}, x_{0}\right)$-orbitally complete, the Cauchy sequence $\left\{y_{2 n}\right\}$ has a limit $u$ in $f_{i}(X)$ for each $i \in \mathbb{N}$. By condition (3.4), we have

$$
0=\lim _{n \rightarrow \infty} d\left(y_{2 n}, y_{2 n+1}\right)=\lim _{n \rightarrow \infty} d\left(u, y_{2 n+1}\right)
$$

and so $\left\{y_{2 n+1}\right\}$ also converges to $u$.

Hence there is a point $z \in X$ such that $f_{i} z=u$. By condition (3.2), for any even $i \in \mathbb{N}$,

$$
\begin{aligned}
D\left(f_{i} z, S z\right) \leq & d\left(f_{i} z, y_{2 n+1}\right)+D\left(y_{2 n+1}, S z\right) \\
\leq & d\left(f_{i} z, y_{2 n+1}\right)+H\left(S z, T x_{2 n+1}\right) \\
\leq & d\left(f_{i} z, y_{2 n+1}\right)+\phi\left(\operatorname { m a x } \left(D\left(f_{i} z, S z\right), D\left(f_{2 n+2} x_{2 n+1}, T x_{2 n+1}\right),\right.\right. \\
& \left.\left.D\left(f_{i} z, T x_{2 n+1}\right), D\left(f_{2 n+2} x_{2 n+1}, S z\right), d\left(f_{i} z, f_{2 n+2} x_{2 n+1}\right)\right)\right) . \\
\leq & d\left(f_{i} z, y_{2 n+1}\right)+\phi\left(\operatorname { m a x } \left(D\left(f_{i} z, S z\right), D\left(y_{2 n+2}, T x_{2 n+1}\right),\right.\right. \\
& \left.\left.D\left(f_{i} z, T x_{2 n+1}\right), D\left(y_{2 n+2}, S z\right), d\left(f_{i} z, y_{2 n+2}\right)\right)\right) . \\
\leq & d\left(f_{i} z, y_{2 n+1}\right)+\phi\left(\operatorname { m a x } \left(D\left(f_{i} z, S z\right), d\left(y_{2 n+2}, y_{2 n+1}\right),\right.\right. \\
& \left.\left.d\left(f_{i} z, y_{2 n+1}\right), d\left(y_{2 n+2}, f_{i} z\right)+D\left(f_{i} z, S z\right), d\left(f_{i} z, y_{2 n+2}\right)\right)\right) .
\end{aligned}
$$

Letting $n \rightarrow \infty$, this inequality yields

$$
D\left(f_{i} z, S z\right) \leq \phi\left(\max \left(D\left(f_{i} z, S z\right) 0,0, D\left(f_{i} z, S z\right), 0\right)\right) .
$$

If $f_{i} z \notin S z$ for any even $i \in \mathbb{N}$, then $D\left(f_{i} z, S z\right)>0$ and the above inequality implies

$$
D\left(f_{i} z, S z\right) \leq \phi\left(D\left(f_{i} z, S z\right)\right)<D\left(f_{i} z, S z\right),
$$

which is a contradiction.

Hence $f_{i} z \in S z$ for every even $i \in \mathbb{N}$ since every proximal set is closed. Similarly, we have $f_{i} z \in T z$ for any odd $i \in \mathbb{N}$. Therefore, $z$ is a coincidence point of $f_{i}, S$ and $T$ for each $i \in \mathbb{N}$.

(2) If for any even $i \in \mathbb{N}, u=f_{i} z$ is a fixed point of $f_{i}$, then $u=f_{i} u=$ $f_{i} f_{i} z \in f_{i} S z$. If $f_{i}$ is weakly commuting with $S$ at $z$, then $f_{i} S z=S f_{i} z$ since 
$f_{i} z \in S z$. Therefore, we have $u \in f_{i} S z=S f_{i} z=S u$; that is, $u$ is a fixed point of $S$.

We can also prove (3) by the same techniques, and by (2) and (3), we have (4).

Since (2.3) implies (3.2), Theorem 3.1 with $S=T$ and $f_{i}=I_{X}$ (the identity mapping on $X$ ) improves slightly Theorem 2.1. Theorem 3.1 remains true when we replace $P_{x}(X)$ by $C L(X)$.

Replacing the condition (3.1) of Theorem 3.1 by orbital regularity, we have the following:

THEOREM 3.2. Let $S$ and $T$ be multi-valued mappings from a metric space $X$ into $P_{x}(X)$ and let $\left\{f_{i}\right\}$ be the family of all continuous mappings from $X$ into itself such that the conditions (3.2) holds,

$$
\phi(t)<t \text { for each } t>0 \text { and some } \phi \in \mathscr{F},
$$

(3.7) for some $x_{0} \in X$, and each $i \in \mathbb{N}$, there exist sequences $\left\{x_{n}\right\}$ and $\left\{y_{n}\right\}$ in $X$ such that the orbit $0_{f_{i}}\left(x_{0}\right)$ is regular, the pair $(S, T)$ is asymptotically regular at $x_{0}$ and $f(X)$ is $\left(S, T ; f_{i}, x_{0}\right)$-orbitally complete. Then we have:

(1) for each $i \in \mathbb{N}, f_{i}, S$ and $T$ have a coincidence point in $X$,

(2) if the limit of $0_{f_{i}}\left(x_{0}\right)$ is a fixed point of $f_{i}$, then the conclusions (2) and (3) of Theorem 3.1 are also true.

We remark that Theorem 3.2 with $S=T$ and $f=f_{i}$ for each $i \in \mathbb{N}$ is Theorem 2 in [16].

It is well known that if $S$ is a multi-valued mapping from $X$ into $C(X)$, then for every $x, y \in X$ and $u \in S x$, there exists a point $v \in S y$ such that

$$
d(u, v) \leq H(S x, S y) .
$$

Hence, if $S$ and $T$ are multi-valued mappings from $X$ into $C L(X)$, then orbital regularity in Theorem 3.2 can be dropped.

THEOREM 3.3. Let $S$ and $T$ be multi-valued mappings from a metric space $X$ into $C(X)$ and let $\left\{f_{i}\right\}$ be the family of continuous mappings fro $X$ into itself such that the conditions (3.1), (3.2), (3.4), (3.5) and (3.6) hold. Then all the conclusions of Theorem 3.2 are also true. 
Consider the following condition:

(3.8) $H(S x, T y) \leq$

$$
\phi\left(\operatorname { m a x } \left(D\left(f_{i} x, S x\right), D\left(f_{j} y, T y\right), D\left(f_{i} x, T y\right), D\left(f_{j} y, S x\right), d\left(f_{i} x, f_{j}(y)\right)\right.\right.
$$

for each $x, y \in X, i, j \in \mathbb{N}, i \neq j$, and $\phi \in \mathscr{F}$.

In Theorem 3.1, if we replace (3.2) by (3.8), the asymptotic regularity of $(S, T),(3.4)$, is not needed.

In fact, we have the following:

THEOREM 3.4. Let $S$ and $T$ be multi-valued mappings from a metric space $X$ into $P_{x}(X)$ and let $\left\{f_{i}\right\}$ be the family of continuous mappings from $X$ into itself such that the conditions (3.1), (3.5) and (3.8) hold. Then all the conclusions of Theorem 3.1 are also true.

In Theorems 3.1 and 3.3, taking $f=f_{i}$ on $X$ for each $i \in \mathbb{N}$ and defining $\phi(t)=q t, 0<q<1$, we have the following:

COROLLARY 3.5. Let $S$ and $T$ be multi-valued mappings from a metric space into $P_{x}(X)$ and let $f$ be a continuous mapping from $X$ into itself such that the condition (3.4) holds, as well as

$$
S(X) \cup T(X) \subseteq f(X) ;
$$

(3.10) $H(S x, T y) \leq$

$$
\begin{gathered}
q \max (D(f x, S x), D(f y, T y), D(f x, T y), D(f y, S x), d(f x, f y)) \\
\text { for each } x, y \in X \text { and for some } 0<q<1,
\end{gathered}
$$

$$
f(X) \text { is }\left(S, T, f, x_{0}\right) \text {-orbitally complete, and }
$$

$f$ is weakly commuting with each of $S$ and $T$.

Then $f, S$ and $T$ have a common fixed point in $X$.

COROLLARY 3.6. Let $S$ and $T$ be multi-valued mappings from a metric space $X$ into $P_{x}(X)$ and let $f$ be a continuous mapping from $X$ into itself such that the conditions (3.9) and (3.12) hold, and

(3.13) $H(S x, T y) \leq$

$$
\begin{gathered}
q \max \left(D(f x, S x), D(f y, T y), \frac{1}{2}(D(f y, S x)+D(f x, T y)), d(f x, f y)\right) \\
\text { for each } x, y \in X \text { and for some } 0<q<1 .
\end{gathered}
$$

Then $f, S$ and $T$ have a common fixed point in $X$. 
The following theorem is an extension of a recent result due to Kubiak [9]:

THEOREM 3.7. Let $S, T$ be multi-valued mappings from a metric space $X$ into $P_{x}(f(X) \cap g(X))$ and $f, g$ be continuous mappings from $X$ into itself such that the conditions (3.12) and (3.13) hold. Then $f, g, S$ and $T$ have a common fixed point in $X$.

REMARK. Our results extend some theorems of Hadzic [1] and Sessa et al. [20] to the version of hybrid contraction mappings.

\section{Some related results}

Let $X$ be a reflexive Banach space and $W C(X)$ denote the family of nonempty weakly compact subsets of $X$. Note that every non-empty weakly compact subset of a reflexive Banach space is proximinal [21] and so closed.

We need the following lemma for our main theorems:

LEMMA 4.1. ([10]) Let $f \in \mathscr{F}$ and $t_{0}>0$. If $t_{n+1} \leq f\left(t_{n}\right)$ for all $n \in \mathbb{N}$, then the sequence $\left\{t_{n}\right\}$ converges to 0 .

The following theorem is an extension of a result due to Kaneko [7]:

THEOREM 4.2. Let $X$ be a reflexive Banach space and let $\left\{S_{n}\right\},\left\{T_{n}\right\}$ be sequences of mappings from $X$ into $W C(X)$ such that

$$
\begin{aligned}
& H\left(S_{m} x, T_{n} y\right) \leq \\
& \phi\left(\max \left(D\left(x, S_{m} x\right), D\left(y, T_{n} y\right), \frac{1}{2}\left(D\left(x, T_{n} y\right)+D\left(y, S_{m} x\right)\right), d(x, y)\right)\right) \\
& \text { for each } x, y \in X \text { and for some } \phi \in \mathscr{F} .
\end{aligned}
$$

Then there exists a point $z \in X$ such that $z \in S_{m} z \cap T_{n} z$ for each $m, n \in \mathbb{N}$.

PROOF. First, we assume that $h=0$. Let $x_{0}=X$ be an arbitrary point. Then there exists a point $x_{1} \in S_{1} x_{0}$ such that $d\left(x_{0}, x_{1}\right)=D\left(x_{0}, S x_{0}\right)$. This is possible because $S_{1} x_{0}$ is a non-empty weakly compact subset of a reflexive Banach space and so is proximinial.

Now, for all $n \in \mathbb{N}$, we have $D\left(x_{1}, T_{n} x_{1}\right) \leq H\left(S_{1} x_{0}, T_{n} x_{1}\right)=0$ and hence we have $x_{1} \in T_{n} x_{1}$. Similarly, for all $n \in \mathbb{N}$,

$$
D\left(x_{1}, S_{1} x_{1}\right) \leq H\left(T_{n} x_{1}, S_{1} x_{1}\right)=0 \quad \text { yields } x_{1} \in S_{n} x_{1} .
$$


Thus $x_{1}$ is a common fixed point of the two equations $\left\{S_{n}\right\}$ and $\left\{T_{n}\right\}$.

Next, we assume that $h \neq 0$. Let $x_{0} \in X$ be arbitrary but fixed.

Construct a sequence $\left\{x_{n}\right\}$ in $X$ such that

$$
\begin{gathered}
x_{2 n-1} \in S_{n} x_{2 n-2}, \quad x_{2 n} \in T_{n} x_{2 n-1}, \\
d\left(x_{2 n-2}, x_{2 n-1}\right)=D\left(x_{2 n-2}, S_{n} x_{2 n-2}\right), \quad \text { and } \\
d\left(x_{2 n-1}, x_{2 n}\right)=D\left(x_{2 n-1}, T_{n} x_{2 n}\right) .
\end{gathered}
$$

The existence of such a sequence in $X$ is guaranteed by the proximinality of $S_{m}(x)$ and $T_{n}(x)$ for each $m, n \in \mathbb{N}$.

Now, suppose that $x_{n}=x_{n+1}$ for some $n \in \mathbb{N}$. If $n \in \mathbb{N}$ is even, then we have $x_{2 n} \in S_{n+1} x_{2 n}$.

Further, for each $m \in \mathbb{N}$, we have

$$
\begin{aligned}
D\left(x_{2 n}, T_{m} x_{2 n}\right) \leq & H\left(S_{n+1} x_{2 n}, T_{m} x_{2 n}\right) \\
\leq & \phi\left(\operatorname { m a x } \left(D\left(x_{2 n}, S_{n+1} x_{2 n}\right), D\left(x_{2 n}, T_{m} x_{2 n}\right),\right.\right. \\
& \left.\left.\quad \frac{1}{2}\left(D\left(x_{2 n}, T_{m} x_{2 n}\right)+D\left(x_{2 n}, S_{n+1} x_{2 n}\right)\right), d\left(x_{2 n} x_{2 n}\right)\right)\right) \\
= & \phi\left(D\left(x_{2 n}, T_{m} x_{2 n}\right)\right) .
\end{aligned}
$$

If $D\left(x_{2 n}, T_{m} x_{2 n}\right)>0$, then it follows from the above inequality that

$$
D\left(x_{2 n}, T_{m} x_{2 n}\right)<D\left(x_{2 n}, T_{m} x_{2 n}\right),
$$

which is a contradiction.

Thus, we have $x_{2 n} \in T_{m} x_{2 n}$ for each $m \in \mathbb{N}$.

Similarly, $D\left(x_{2 n}, S_{m} x_{2 n}\right) \leq H\left(S_{m} x_{2 n}, T x_{2 n}\right) \leq \phi\left(D\left(x_{2 n}, S_{m} x_{2 n}\right)\right)$ gives $x_{2 n} \in$ $S_{m} x_{2 n}$ for each $m \in \mathbb{N}$.

For an odd number $n \in \mathbb{N}$, we have also the same results.

Therefore, in each case, we have a common fixed point for the sequences $\left\{S_{m}\right\}$ and $\left\{T_{n}\right\}$.

Suppose now that $x_{n} \neq x_{n+1}$ for all $n \in \mathbb{N}$. We shall show that $\left\{x_{n}\right\}$ is a Cauchy sequence in $X$. Let us first observe that

$$
\begin{aligned}
d\left(x_{2 n}, x_{2 n+1}\right)= & D\left(x_{2 n}, S_{n+1} x_{2 n}\right) \\
\leq & H\left(T_{n} x_{2 n-1}, S_{n+1} x_{2 n}\right) \\
\leq & \phi\left(\operatorname { m a x } \left(D\left(x_{2 n-1}, S_{n+1} x_{2 n}\right), D\left(x_{2 n-1}, T_{n} x_{2 n-1}\right),\right.\right. \\
& \left.\left.\quad \frac{1}{2}\left(D\left(x_{2 n}, T_{n} x_{2 n-1}\right)+D\left(x_{2 n-1}, S_{n+1} x_{2 n}\right)\right), d\left(x_{2 n}, x_{2 n-1}\right)\right)\right)
\end{aligned}
$$




$$
\begin{gathered}
=\phi\left(\max \left(d\left(x_{2 n-1}, x_{2 n}\right), d\left(x_{2 n}, x_{2 n+1}\right), \frac{1}{2} d\left(x_{2 n-1}, x_{2 n+1}\right)\right)\right) \\
\leq \phi\left(\operatorname { m a x } \left(d\left(x_{2 n-1}, x_{2 n}\right), d\left(x_{2 n}, x_{2 n+1}\right),\right.\right. \\
\left.\left.\quad \frac{1}{2}\left(d\left(x_{2 n-1}, x_{2 n}\right)+d\left(x_{2 n}, x_{2 n+1}\right)\right)\right)\right) .
\end{gathered}
$$

If $d\left(x_{2 n-1}, x_{2 n}\right)<d\left(x_{2 n}, x_{2 n+1}\right)$, then the above inequality yields

$$
d\left(x_{2 n}, x_{2 n+1}\right) \leq \phi\left(d\left(x_{2 n}, x_{2 n+1}\right)\right)<d\left(x_{2 n}, x_{2 n+1}\right),
$$

which is a contradiction. Therefore, we get

$$
d\left(x_{2 n}, x_{2 n+1}\right) \leq \phi\left(d\left(x_{2 n-1}, x_{2 n}\right)\right) .
$$

Similarly, we can show that

$$
d\left(x_{2 n+1}, x_{2 n+2}\right) \leq \phi\left(d\left(x_{2 n}, x_{2 n+1}\right)\right)
$$

It follows from the above relations that

$$
d\left(x_{n}, x_{n+1}\right) \leq \phi\left(d\left(x_{n}, x_{n-1}\right)\right) .
$$

By Lemma 4.1, we have $\lim _{n \rightarrow \infty} d\left(x_{n}, x_{n+1}\right)=0$, which yields that $\left\{x_{n}\right\}$ is a Cauchy sequence in $X$. Since $X$ is a Banach space, this Cauchy sequence converges to a point in $X$. Let $\lim _{n \rightarrow \infty} x_{n}=z$. Then we have

$$
\begin{aligned}
D\left(x_{2 n-1}, T_{m} z\right) \leq H\left(S_{n} x_{2 n-2}, T_{m} z\right) & \\
\leq & \phi\left(\operatorname { m a x } \left(D\left(x_{2 n-2}, S_{n} x_{2 n-2}\right), D\left(z, T_{m} z\right),\right.\right. \\
& \left.\left.\quad \frac{1}{2}\left(D\left(x_{2 n-2}, T_{m} z\right)+D\left(z, S_{n} x_{2 n-2}\right)\right), d\left(x_{2 n-2}, z\right)\right)\right) \\
& \leq \phi\left(\operatorname { m a x } \left(d\left(x_{2 n-2}, x_{2 n-1}\right), D\left(z, T_{m} z\right),\right.\right. \\
& \left.\left.\frac{1}{2}\left(D\left(x_{2 n-2}, T_{m} z\right)+d\left(z, x_{2 n-1}\right)\right), d\left(x_{2 n-2}, z\right)\right)\right) .
\end{aligned}
$$

Letting $n \rightarrow \infty$, the above inequality yields

$$
D\left(z, T_{m} z\right)=0 \text {. }
$$

Since $T_{m} z$ is closed, $z \in T_{m} z$ for all $m \in \mathbb{N}$.

Similarly, we have also $z \in S_{m} z$ for all $m \in \mathbb{N}$.

Therefore, $z$ is a common fixed point of the sequence $\left\{S_{n}\right\}$ and $\left\{T_{n}\right\}$. This completes the proof. 
Let $f, g$ be single-valued mappings from a metric space $X$ into itself. Recall that the mappings $f$ and $g$ are said to be weakly commuting [17] if $d(f g x, g f x) \leq d(f x, g x)$ for any $x \in X$.

Clearly two commuting mappings [4] (that is, $f g x=g f x$ for any $x \in X$ ) are weakly commuting but the converse is not true.

EXAMPLE. Let $X=[0,1]$ with the Euclidean metric $d$ and let $f, g X \rightarrow X$ be defined by $f(x)=2 x /(x+1)$ and $g(x)=x /(2 x+1)$ for all $x \in X$. Then the mappings $f$ and $g$ are weakly commuting but not commuting.

Some fixed point theorems for commuting and weakly commuting mappings may be found in [3-5] and [14-18, 20].

COROLLARY 4.3. Let $f, g, S$ and $T$ be single-valued mappings from a complete metric space $X$ into itself such that

$$
\begin{array}{r}
d(f x, g y) \leq \\
\varphi\left(\max \left(d(f x, S x), d(g y, T y), \frac{1}{2}(d(f x, T y)+d(g y, S x)), d(S x, T y)\right)\right) \\
\text { for each } x, y \in X \text { and for some } \varphi \varepsilon \mathscr{F},
\end{array}
$$

$$
(f, S) \text { and }(g, T) \text { are weakly commuting pairs. }
$$

Then $f, g, S$ and $T$ have a unique common fixed point in $X$.

Proof. Define two mappings $A, B$ by $A x=\{f x\}$ and $B x=\{g x\}$ for any $x \in X$. Then $(A, S)$ and $(B, T)$ are weakly commuting pairs. Now in view of Theorem 4.2, there exists a point $z \in X$ such that $S z \in A z$ and $T z \in B z$, which yields $S z=f z$ And $T z=g z$. Substituting in (4.2), we obtain

$$
\begin{aligned}
d(f z, g z) & \leq \varphi\left(\max \left\{d(f z, S z), d(g z, T z), \frac{1}{2}[d(f x, T z)+d(g z, S z)], d(S z, T z)\right\}\right) \\
& =\varphi(\max \{0,0, d(f x, g z), d(f z, g z)\})=\phi(d(f z, g z)) .
\end{aligned}
$$

If $f z \neq g z$, then we obtain the contradiction $d(f z, g z)<d(f z, g z)$. Therefore $z$ is a common coincidence point of $f, g, S$, and $T$.

We shall now show that $f z$ is a common fixed point of $f, g, S$ and $T$.

Since $f$ and $S$ weakly commute, $d(S f z, f S z) \leq d(S x, f z)=0$ and $S f z=$ $f S z$. But $S z=f z$. Therefore $S f z=f S z=f^{2} z$ and $f z$ is a common coincidence point of $f$ and $S$. 
Since $g$ and $T$ weakly commute, $d(g t Z, T g z) \leq d(T z, g z)=0$ and $g T z=$ $T g z$. Since $g z=T z$ we obtain $T g z=g T z=g^{2} z$ and $g z$ is a common coincidence point of $f, g, S$ and $T$. Since $f z=g z, f z$ is a common coincidence point of $f, g, S$ and $T$.

From (4.2), we obtain

$$
\begin{aligned}
d(f z, g f z) \leq & \varphi(\max \{d(f z, S z), d(g f z, T f z), \\
& \left.\left.\frac{1}{2}[d(f z, T f z)+d(g f z, S z)], d(S z, T f z)\right\}\right) \\
& =\varphi(\max \{0,0, d(f z, g f z), d(f z, g f z)\}) \\
& =\varphi(d(f z, g f z)),
\end{aligned}
$$

which forces $f z=g f z$ and $f z$ is a fixed point of $g$. From $T g z=g^{2} z$ and $f z=g z$, it follows that $f z$ is also a fixed point of $T$.

If we can show that $f z$ is also a fixed point of $f$, then, from $S f z=f^{2} z=f z$, it follow that $f z$ is a also a fixed point of $S$. So consider

$$
\begin{aligned}
d(f f z, f z) & =d(f f z, g z) \\
& \leq \varphi(\max \{d(f f z, S f z), d(g z, T z), \\
& \left.\left.\quad \frac{1}{2}[d(f f z, T z)+d(g z, S f z)], d(S f z, T z)\right\}\right) \\
& =\varphi(\max \{0,0, d(f f z, f z), d(f f z, f z)\}) \\
& =\varphi(d(f f z, f z)),
\end{aligned}
$$

which implies that $f f z=f z$.

We shall now show uniqueness. Suppose that $u$ and $v$ are common fixed points of $f, g, S$ and $T$. Then, from (4.2),

$$
\begin{aligned}
d(u, v)= & d(f u, g v) \\
\leq & \phi(\max \{d(f u, S u), d(g v, T v), \\
& \left.\left.\quad \frac{1}{2}[d(f u, T v)+d(g v, S u)], d(S u, T v)\right\}\right) \\
= & \phi(\max \{0,0, d(u, v), d(u, v)\}) \\
= & \phi(d(u, v)),
\end{aligned}
$$

which implies that $u=v$. 
COROLLARY 4.4. [20] Let $S$ and $T$ be two continuous mappings from a complete metric space $X$ into itself. Then $S$ and $T$ have a common fixed point in $X$ if and only if there are two self-mappings $A$ and $B$ on $X$ such that

$$
\begin{array}{r}
\varphi\left(\max \left(d(S x, A x), d(T y, B y), \frac{1}{2}(d(S x, B y)+d(T y, A x)), d(S x, T y)\right)\right) \\
\text { for each } x, y \in X \text { and for some } \phi \in \mathscr{F},
\end{array}
$$

$$
(A, S) \text { and }(B, T) \text { are weakly commuting pairs. }
$$

Further, $z$ is the unique common fixed point of $A, B, S$ andT.

THEOREM 4.5. Let $X$ be a compact metric space and let $S, T$ be multi-valued mappings from $X$ into $P_{x}(X)$ such that either $S$ or $T$ is continuous,

$$
\begin{aligned}
& H(S x, T y) \leq \\
& \phi\left(\max \left(d(x, S x), d(y, T y), \frac{1}{2}(d(x, T y)+d(y, S x)), d(x, y)\right)\right) \\
& \text { for each } x, y \in X, x \neq y,
\end{aligned}
$$

and for some $\phi \in \mathscr{F}$. Then either $S$ or $T$ has a fixed point in $X$.

Proof. Suppose that $S$ is continuous. Define $\varphi X \rightarrow \mathbb{R}$ by $\varphi(x)=D(x, S x)$ for all $x \in X$. Then $\varphi$ is continuous on $X$. Since $X$ is compact, there exists a point $x_{0} \in X$ such that $\varphi\left(x_{0}\right)=\min \{\varphi(x): x \in X\}$. Now, choose $x_{1} \in S x_{0}$ such that $d\left(x_{0}, x_{1}\right)=D\left(x_{0}, S x_{0}\right)$. This is possible because $S x_{0}$ is proximimal. Similarly, we can choose $x_{2} \in T x_{1}$ and $x_{3} \in S x_{2}$ such that

$$
d\left(x_{1}, x_{2}\right)=D\left(x_{1}, T x_{1}\right) \quad \text { and } \quad d\left(x_{2}, x_{3}\right)=D\left(x_{2}, S x_{2}\right) .
$$

Let us suppose that $d\left(x_{0}, S x_{0}\right)>0$ and $d\left(x_{1}, T_{1} x\right)>0$. Then we have

$$
\begin{aligned}
d\left(x_{1}, x_{2}\right) \leq & H\left(S x_{0}, T x_{1}\right) \\
< & \phi\left(\operatorname { m a x } \left(D\left(x_{0}, S x_{0}\right), D\left(x_{1}, T x_{1}\right),\right.\right. \\
& \left.\left.\quad \frac{1}{2}\left(D\left(x_{0}, T x_{1}\right)+D\left(x_{1}, S x_{0}\right)\right), d\left(x_{0}, x_{1}\right)\right)\right) \\
\leq & \phi\left(\max \left(d\left(x_{1}, x_{2}\right), \frac{1}{2} d\left(x_{0}, x_{2}\right), d\left(x_{0}, x_{1}\right)\right)\right),
\end{aligned}
$$

which yield $d\left(x_{1}, x_{2}\right)<d\left(x_{0}, x_{1}\right)$. 
Similarly, we have also $d\left(x_{2}, x_{3}\right)<d\left(x_{1}, x_{2}\right)$. Therefore, we have $d\left(x_{2}, x_{3}\right)<d\left(x_{0}, x_{1}\right)=\varphi\left(x_{0}\right)$, which is a contradiction to the minimality of $\varphi\left(x_{0}\right)$. Hence either $D\left(x_{0}, S x_{0}\right)=0$ or $D\left(x_{1}, T x_{1}\right)=0$. Since the proximal sets $S x_{0}$ and $T x_{1}$ are closed, it follows that either $x_{0} \in S x_{0}$ or $x_{1} \in T x_{1}$. This completes the proof.

\section{Acknowledgement}

The authors thank the learned referee for several useful comments.

\section{References}

[1] O. Hadzic, 'Common fixed point theorems for family of mappings in complete metric spaces', Math. Japon. 29 (1984), 127-134.

[2] S. Itoh and W. Takahashi, 'Single-valued mappings, multi-valued mappings and fixed point theorems', J. Math. Anal. Appl. 59 (1977), 261-263.

[3] G. Jungck, 'Common fixed points for commuting and compatible maps on compacts', to appear.

[4] _- 'Commuting mapping and fixed points', Amer. Math. Monthly 83 (1976), 261-263.

[5] _- 'Periodic and fixed points, and commuting mappings', Proc. Amer. Math. Soc. 76 (1979), 333-338.

[6] H. Kaneko, 'Single-valued and multi-valued f-contractions', Boll. Un. Mat. Ital. A 4 (1985), 29-33.

[7] _ 'A comparison of contractive conditions for multi-valued mappings', Kobe J. Math. 3 (1986), 37-45.

[8] M. S. Khan, 'Common fixed point theorems for multi-valued mappings', Pacific J. Math. 95 (1981), 337-347.

[9] T. Kubiak, 'Fixed point theorems for contractive type multi-valued mapping', Math. Japon. 30 (1985), 89-101.

[10] B. A. Meade and S. P. Singh, 'On common fixed point theorems', Bull. Austral. Math. Soc. 16 (1977), 49-53.

[11] R. N. Mukherjee, 'On fixed points of single-valued and set-valued mappings', J. Indian Acad. Math. 4 (1982), 101-103.

[12] S. B. Nadler, 'Multivalued contraction mappings', Pacific J. Math. 30 (1969), 475-488.

[13] S. A. Naimpally, S. L. Singh and J. H. M. Whitfield, 'Coincidence theorems for hybrid contractions', Math. Nachr. 127 (1986), 177-180.

[14] B. E. Rhoades and S. Sessa, 'Common fixed point theorems for three mappings under a weak commutativity condition', Indian J. Pure Appl. Math. 17 (1986), 47-57.

[15] B. E. Rhoades, S. Sessa, M. S. Khan and M. Swaleh, 'On fixed points of asymptotically regular mappings', J. Austral. Math. Soc. (Series A) 43 (1987), 328-346. 
[16] B. E. Rhoades, S. L. Singh and C. Kulshrestha, 'Coincidence theorems for some multivalued mappings', Internat. J. Math. Sci. 7 (1984), 429-434.

[17] S. Sessa, 'On a weak commutativity condition in fixed point considerations', Publ. Inst. Math. 32 (1982), 149-153.

[18] S. Sessa and B. Fisher, 'On common fixed points of weakly commuting mappings and set-valued mappings', Internat. J. Math. Soc. 9 (1986), 323-329.

[19] S. Sessa, M. S. Khan and M. Imada, 'A common fixed point theorem with a weak commutativity condition', Glas. Mat. Ser. III 21 (1986), 225-235.

[20] S. Sessa, R. N. Mukherjee and T. Som, 'A common fixed point theorem for weakly commuting mappings', Math. Japon. 31 (1986), 235-245.

[21] I. Singer, 'The theory of best approximation and functional analysis', in: CBMS-NSF Regional Conf. Ser. in Appl. Math. 13 (SIAM, Philadelphia, 1974).

[22] R. E. Smithon, 'Fixed points for contractive multifunctions', Proc. Amer. Math. Soc. 27 (1971), 192-194.

[23] Lj. B. Čirič, 'Fixed points for generalized multivalued contractions', Math. Vesnik 9 (1972), 265-272.

[24] R. Wegrzyk, 'Fixed point theorems for multi-valued functions and their applications to functional equations', Dissertatione Math. (Rozprawy Mat.) 201 (1982), 1-28.

Sultan Qaboos University

Department of Mathematics

and Computing

College of Science

P.O. Box 32486

Al-Khod, Muscat

Sultanate of Oman
Gyeongsang National University Jinju 660-701

Korea
Gyeongsang National University

Jinju 660-701

Korea
Aligarh Muslim University Aligarh 202002 India 